\title{
Beyond survival: What are the outcomes that really matter to our patients?
}

\author{
K. A. Mussatto
}

Cardiol Young 2006; 16: 125-127

The publisher apologises for this error. The correct

The above Editorial Comment, which was published in Volume 16, Number 2 (April 2006), the author's name was misspelt. It should have read "Mussatto". reference should therefore be:

Mussatto KA. Beyond survival: What are the outcomes that really matter to our patients? Cardiol Young 2006; 16(2): 125-127. 